\title{
Vibration-Attenuation Controller Design for Uncertain Mechanical Systems with Input Time Delay
}

\author{
Yuanchun Ding, ${ }^{1}$ Falu Weng, ${ }^{2}$ Xiaohua Jiang, ${ }^{1}$ and Minkang Tang ${ }^{1}$ \\ ${ }^{1}$ Jiangxi Key Laboratory of Mining Engineering, Jiangxi University of Science and Technology, Ganzhou, Jiangxi 341000, China \\ ${ }^{2}$ Faculty of Electrical Engineering and Automation, Jiangxi University of Science and Technology, Ganzhou, Jiangxi 341000, China \\ Correspondence should be addressed to Falu Weng; 10932014@zju.edu.cn
}

Received 3 July 2015; Accepted 10 September 2015

Academic Editor: Alba Sofi

Copyright (c) 2016 Yuanchun Ding et al. This is an open access article distributed under the Creative Commons Attribution License, which permits unrestricted use, distribution, and reproduction in any medium, provided the original work is properly cited.

\begin{abstract}
The problems of vibration-attenuation controller design for uncertain mechanical systems with time-varying input delay are of concern in this paper. Firstly, based on matrix transformation, the mechanical system is described as a state-space model. Then, in terms of introducing the linear varying parameters, the uncertain system model is established. Secondly, the LMI-based sufficient conditions for the system to be stabilizable are deduced by utilizing the LMI technique. By solving the obtained LMIs, the controllers are achieved for the closed-loop system to be stable with a prescribed level of disturbance attenuation. Finally, numerical examples are given to show the effectiveness of the proposed theorems.
\end{abstract}

\section{Introduction}

Mechanical systems play an important role in human lives, production, and industrial engineering. Although some new technologies, especially information and computer technologies, are developed recently, mechanical systems are still not replaceable in many engineering fields. Meanwhile, with the development of automatic control theories, mechanical systems become much more stable and effective than before [1-3]. In recent years, many advanced theories in systems and control have been applied to mechanical systems. For example, sliding mode control [1], $H_{\infty}$ control $[3,4]$, optimal design and control $[5,6]$, energy-based control [7], finitetime stabilization [8], and position feedback control [9] have been widely studied and used for control of mechanical systems.

On the other hand, time delay or transportation lag is inevitable in many practical systems, such as chemical processes, long transmission lines, and pneumatic and hydraulic systems [10-12]. For mechanical systems, unavoidable time delays always appear in the controlled channel, particularly in the digital controller as it carries out the calculations associated with complex sophisticated control law and in sensors and actuators hardware such as large hydraulic actuators. Though the delay time may be short, it can nevertheless limit the control performance or even cause the instability of the system when the delay appears in the feedback loop [10]. Furthermore, most of those delays are time-varying because of the perturbance and uncertainties always existing in the practical systems $[13,14]$. Recently, many control strategies have been presented to deal with the control problem for time-delay systems. Such as [15] presented a robust $H_{\infty}$ controller design approach to deal with the vibration-attenuation of uncertain mechanical systems with input delay. Based on LMI technique, the problem of robust active vibration control for a class of electrohydraulic actuated structural systems with time delay in the control input channel and parameter uncertainties appearing in all the mass, damping, and stiffness matrices was investigated in [16]. Based on LMI technique, the delayed $H_{\infty}$ control for an offshore steel jacket platforms subject to external wave force was presented in [17]. Based on Lyapunov theory, the problem of finite-time vibration control of earthquake excited linear structures with input time-delay and saturation was investigated in [12]. Moreover, uncertainty is another problem that need to be considered $[18,19]$. Due to the modeling errors, material properties, 
or environments changing, the description of mechanical systems inevitably contains uncertainties, which can affect the stability and control performance of the mechanical systems significantly. For mechanical systems, the parameter uncertainties and time delay often appear simultaneously, thus, in our investigation, it is important to take both of them into consideration, otherwise the designed controllers will collapse in the practical situations. However, to the best of the authors' knowledge, the uncertain mechanical systems with time-varying input delay still have not been fully investigated, which is the main motivation of this paper.

In this paper, we consider the problems of vibrationattenuation controller design for uncertain mechanical systems with time-varying input delay. The objective of designing controllers is to guarantee the asymptotic stability of closed-loop systems while attenuating disturbance from external excitation. The main contribution of this paper consists in two aspects. First, in terms of introducing the linear varying parameters, the uncertain system model for mechanical systems with time-varying input delay is established. Second, based on Lyapunov stability theory and LMI technique, the LMI-based condition for the system to be stabilizable is deduced. By solving these LMI, the controller is established for the closed-loop system to be stable with the performance $\|z\|_{2}<\gamma\|\omega\|_{2}$. Furthermore, example is given to demonstrate the effectiveness of the proposed theorems.

\section{Problem Formulation and Preliminaries}

The mechanical systems with time-varying input delay are described as $[3,12,15]$

$$
\bar{M} \ddot{\bar{x}}(t)+\bar{C} \dot{\bar{x}}(t)+\bar{K} \bar{x}(t)=\bar{B} u(t-d(t)) .
$$

This model has been widely applied to the description of mechanical and structural systems. Where $\bar{x}(t) \in R^{n}$ is the displacement; $\bar{M} \in R^{n \times n}, \bar{C} \in R^{n \times n}$, and $\bar{K} \in R^{n \times n}$ are the mass, damping, and stiffness matrices, respectively; $\bar{M}$ is assumed to be nonsingular as usual; $\bar{B} \in R^{n \times m}$ is the gain matrix for the state-feedback control input $u(t), d(t)$ is a time-varying delay satisfying $0 \leq d(t) \leq d$, and $d$ is a constant. The state feedback controller consists of the displacement and velocity feedback signals given by

$$
u(t)=F_{d} \bar{x}(t)+F_{v} \dot{\bar{x}}(t),
$$

where $F_{d}, F_{v}$ are the feedback gain matrices for the displacements and velocities, respectively. By defining $x(t)=$ $\left[\bar{x}^{T}(t), \dot{\bar{x}}^{T}(t)\right]^{T}$ and considering an input disturbance signal $\omega(t) \in L_{2}[0, \infty)$ (i.e., with bounded energy) and output signal $Z(t)$, we have the following feedback control systems:

$$
\begin{aligned}
E \dot{x}(t) & =A x(t)+B u(t-d(t))+B_{\omega} \omega(t), \\
Z(t) & =C x(t), \\
u(t) & =F x(t),
\end{aligned}
$$

where $E=\left[\begin{array}{ll}I & 0 \\ 0 & \bar{M}\end{array}\right], A=\left[\begin{array}{cc}0 & I \\ -\bar{K} & -\bar{C}\end{array}\right], B=\left[\begin{array}{c}0 \\ \bar{B}\end{array}\right], B_{\omega}=\left[\begin{array}{c}0 \\ \bar{B}_{\omega}\end{array}\right]$ is the disturbance gain matrix, $C \in R^{q \times 2 n}$ is the output matrix, and $F$ is the state-feedback gain matrix given by $F=\left[\begin{array}{ll}F_{d} & F_{v}\end{array}\right]$.
Remark 1. It is worthy to point out that the input time delay $d(t)$ considered in this paper is not a constant. Thus, the results obtained in this paper should be less conservatism and more fit for using in practical systems than those achievements obtained by the systems with constant time delay (some results about constant time delay can be found in $[12,15-18]$ and those references therein).

By considering the additive uncertainties, the uncertain description of system (3) is written by [20]

$$
\begin{aligned}
E(\alpha) \dot{x}(t) & =A(\alpha) x(t)+B(\alpha) u(t-d(t))+B_{\omega} \omega(t), \\
Z(t) & =C x(t), \\
u(t) & =F x(t)
\end{aligned}
$$

where $E(\alpha)$ is nonsingular, and $E(\alpha), A(\alpha), B(\alpha)$ are in the following convex sets:

$$
[E(\alpha), A(\alpha), B(\alpha)]=\sum_{i=1}^{k} \alpha_{i}\left[E_{i}, A_{i}, B_{i}\right]
$$

with $\sum_{i=1}^{k} \alpha_{i}=1, \alpha_{i} \geq 0, i=1,2, \ldots, k$.

Lemma 2 (see [21]). For any positive definite symmetric constant matrix $\Pi \in R^{n \times n}$, scalars $r_{1}, r_{2}$ satisfying $r_{1}<r_{2}$, a vector function $\omega:\left[r_{1}, r_{2}\right] \rightarrow R^{n}$ such that the integrations concerned are well defined, then

$$
\begin{aligned}
& \left(\int_{r_{1}}^{r_{2}} \omega(s) d s\right)^{T} \Pi\left(\int_{r_{1}}^{r_{2}} \omega(s) d s\right) \\
& \quad \leq\left(r_{2}-r_{1}\right)\left(\int_{r_{1}}^{r_{2}} \omega(s)^{T} \Pi \omega(s) d s\right) .
\end{aligned}
$$

Lemma 3 (see [22]). For any vectors $x, y \in R^{n}$ and any positive definite matrix $L \in R^{n \times n}$, then

$$
-2 x^{T} y \leq x^{T} L^{-1} x+y^{T} L y .
$$

In this paper, the aim is to find a controller gain $F$ such that the closed-loop system has the following properties: (i) asymptotically stable; (ii) for all non-zero $\omega \in L_{2}[0, \infty)$ and the prescribed constant $\gamma>0$, it has the performance $\|z\|_{2}<$ $\gamma\|\omega\|_{2}$.

\section{Main Results}

In this section, we give a solution to the stabilization and robust stabilization problems formulated previously, by using LMI technique.

Theorem 4. The system (3) is asymptotically stabilizable with time delay $d(t)$ satisfying $0 \leq d(t) \leq d$ and performance $\|z\|_{2}<\gamma\|\omega\|_{2}$ for all nonzero $\omega \in L_{2}[0, \infty)$, and constant 
$\gamma>0$, if there exist positive definite symmetric matrices $P, R$, $Q$, matrices $V, Y_{1}, Y_{2}, Y_{3}, Y_{4}, Z$, positive scalar $\mu$, and scalars $\beta_{1}, \beta_{2}$ satisfying the following LMI:

$$
\Psi=\left[\begin{array}{cc}
\Xi_{1} & d Y \\
* & -d Q
\end{array}\right]<0,
$$

where

$$
\begin{aligned}
& \Xi_{1} \\
& =\left[\begin{array}{cccc}
\Xi_{11} & \Xi_{12} & P-V E^{T}+\beta_{2} A V^{T}+Y_{3}^{T} & V C^{T}+Y_{4}^{T} \\
* & \Xi_{22} & \beta_{1} V E^{T}+\beta_{2} B Z-Y_{3}^{T} & \beta_{1} V C^{T}-Y_{4}^{T} \\
* & * & d^{2} Q-\beta_{2} V E^{T}-\beta_{2} E V^{T} & \beta_{2} V C^{T} \\
* & * & * & -\gamma^{2} I
\end{array}\right], \\
& \Xi_{11}=R+B_{\omega} B_{\omega}^{T}+V A^{T}+A V^{T}+Y_{1}^{T}+Y_{1}, \\
& \Xi_{12}=Z^{T} B^{T}+\beta_{1} A V^{T}+Y_{2}^{T}-Y_{1}, \\
& \Xi_{22}=-(1-\mu) R+\beta_{1} Z^{T} B^{T}+\beta_{1} B Z-Y_{2}^{T}-Y_{2} \text {, } \\
& Y=\left[\begin{array}{llll}
Y_{1}^{T} & Y_{2}^{T} & Y_{3}^{T} & Y_{4}^{T}
\end{array}\right]^{T} .
\end{aligned}
$$

Moreover, a state-feedback controller is described as $F=Z V^{-T}$.

Proof. Consider the system without uncertainties. Substituting the control law $u(t)=F x(t)$ into the system (3) results in the following closed-loop system:

$$
\begin{aligned}
E \dot{x}(t) & =A x(t)+B F x(t-d(t))+B_{\omega} \omega(t), \\
Z(t) & =C x(t) .
\end{aligned}
$$

Noting that the solutions to $\operatorname{det}\left(S E-A-B K e^{-s \tau}\right)=0$ are the same as those of $\operatorname{det}\left(S E^{T}-A^{T}-K^{T} B^{T} e^{-s \tau}\right)=0$, and $\left\|T_{z \omega}\right\|_{\infty}=\left\|T_{z \omega}^{T}\right\|_{\infty}$, as long as the $H_{\infty}$ performance and stability are concerned, we can consider the following system instead of system (10):

$$
\begin{aligned}
E^{T} \dot{x}(t) & =A^{T} x(t)+F^{T} B^{T} x(t-\tau)+C^{T} \omega(t), \\
Z(t) & =B_{\omega}^{T} x(t) .
\end{aligned}
$$

Choose a Lyapunov-Krasovskii functional candidate as

$$
\begin{aligned}
V(t)= & x^{T}(t) P x(t)+\int_{t-d(t)}^{t} x^{T}(s) R x(s) d s \\
& +d \int_{-d}^{0} \int_{t+\theta}^{t} \dot{x}^{T}(s) Q \dot{x}(s) d s d \theta .
\end{aligned}
$$

By Lemma 2, the time derivative of $V(t)$ along the solution of (11) is given by

$$
\begin{aligned}
\dot{V}(t) \leq & \dot{x}^{T}(t) P x(t)+x^{T}(t) P \dot{x}(t)+x^{T}(t) R x(t) \\
& -(1-\mu) x^{T}(t-d(t)) R x(t-d(t)) \\
& +d^{2} \dot{x}^{T}(t) Q \dot{x}(t) \\
& -\int_{t-d}^{t} \dot{x}^{T}(\theta) d \theta Q \int_{t-d}^{t} \dot{x}(\theta) d \theta .
\end{aligned}
$$

For any matrix $Y=\left[\begin{array}{lllll}Y_{1}^{T} & Y_{2}^{T} & Y_{3}^{T} & Y_{4}^{T}\end{array}\right]^{T}$ with appropriate dimension, the following equation holds:

$$
2 \eta^{T}(t) Y\left(x(t)-x(t-d)-\int_{t-d}^{t} \dot{x}(\theta) d \theta\right)=0
$$

where $\eta(t)=\left[\begin{array}{lll}x(t)^{T} & x(t-d(t))^{T} \quad \dot{x}(t)^{T} \quad \omega(t)^{T}\end{array}\right]^{T}$. For any matrix $V$ with appropriate dimension, the following equation holds:

$$
\begin{aligned}
& 2\left(x^{T}(t)+\beta_{1} x^{T}(t-d(t))+\beta_{2} \dot{x}^{T}(t)\right) \\
& \quad \cdot V\left(A^{T} x(t)+F^{T} B^{T} x(t-\tau)+C^{T} \omega(t)-E^{T} \dot{x}(t)\right) \\
& \quad=0 .
\end{aligned}
$$

By Lemma 3, we have

$$
\begin{aligned}
-2 \eta^{T}(t) Y \int_{t-d}^{t} \dot{x}(\theta) d \theta \\
\leq d \eta^{T}(t) Y^{T} Q^{-1} Y \eta(t) \\
\quad+\int_{t-d}^{t} \dot{x}^{T}(\theta) d \theta Q \int_{t-d}^{t} \dot{x}(\theta) d \theta .
\end{aligned}
$$

Next, we will establish the $\|z\|_{2}<\gamma\|\omega\|_{2}$ performance for the system under zero initial condition, that is, $\Phi(t)=0, \forall t \in$ $[-\tau, 0]$, and $\left.V(t)\right|_{t=0}=0$. Consider the following index:

$$
J=\int_{0}^{\infty}\left[Z^{T}(t) Z(t)-\gamma^{2} \omega^{T}(t) \omega(t)\right] d t
$$

Then, for any nonzero $\omega(t) \in L_{2}[0, \infty)$, there holds

$$
\begin{aligned}
J \leq & \int_{0}^{\infty}\left(Z^{T}(t) Z(t)-\gamma^{2} \omega^{T}(t) \omega(t)\right) d t+\left.V(t)\right|_{t=\infty} \\
& -\left.V(t)\right|_{t=0} \\
= & \int_{0}^{\infty}\left(Z^{T}(t) Z(t)-\gamma^{2} \omega^{T}(t) \omega(t)+\dot{V}(t)\right) d t .
\end{aligned}
$$

Choosing $F V^{T}=Z$ and noting (13)-(18), after some algebraic manipulations, we obtain

$$
\begin{gathered}
Z^{T}(t) Z(t)-\gamma^{2} \omega^{T}(t) \omega(t)+\dot{V}(t) \\
\leq \eta^{T}(t)\left(\Xi_{1}+d Y Q Y^{T}\right) \eta(t) .
\end{gathered}
$$


Then, if $\Xi_{1}+d Y Q Y^{T}<0$, we have $\int_{0}^{\infty}\left[Z^{T}(t) Z(t)-\right.$ $\left.\gamma^{2} \omega^{T}(t) \omega(t)+\dot{V}(t)\right] d t<0$. Thus, for any nonzero $\omega(t) \epsilon$ $L_{2}[0, \infty)$, we obtain $J<0$ and $\|z\|_{2}<\gamma\|\omega\|_{2}$. If $\Xi_{1}+$ $d Y Q Y^{T}<0$ and $\omega(t) \equiv 0$, we can easily obtain $\dot{V}(t)<0$, and the asymptotic stabilizability of system (11) is established. According to the Schur compliment, we can obtain $\Xi_{1}+$ $d Y Q Y^{T}<0$ from (8). This completes the proof.

Remark 5. Note that the matrix inequality (8) in Theorem 4 is actually an LMI when the scalars $\beta_{1}$ and $\beta_{2}$ are given in advance; thus, the solutions of Theorem 4 can be easily obtained by the powerful LMI toolbox in MATLAb. Furthermore, the two scalars $\beta_{1}$ and $\beta_{2}$ supply an additional degree of freedom for the feasibility of LMI (8). For example, the scalars $\beta_{1}$ and $\beta_{2}$ can all be set to 1 , initially. If LMI (8) is infeasible, a possible solution may be searched by tuning $\beta_{1}$ and $\beta_{2}$ or by iterating over $\beta_{1}$ and $\beta_{2}$.

Theorem 6. The system (4) is robustly stabilizable with time delay $d(t)$ satisfying $0 \leq d(t) \leq d$ and performance $\|z\|_{2}<$ $\gamma\|\omega\|_{2}$ for all nonzero $\omega \in L_{2}[0, \infty)$, and constant $\gamma>0$, if there exist positive definite symmetric matrices $P, R, Q$, matrices $V, Y_{1}, Y_{2}, Y_{3}, Y_{4}, Z$, positive scalar $\mu$, and scalars $\beta_{1}$, $\beta_{2}$ satisfying the following LMIs:

$$
\left[\begin{array}{cc}
\widetilde{\Xi}_{i} & d Y \\
* & -d Q
\end{array}\right]<0, \quad i=1,2, \ldots, k
$$

where

$$
\begin{aligned}
& \widetilde{\Xi}_{i} \\
& =\left[\begin{array}{cccc}
\widetilde{\Xi}_{i 11} & \widetilde{\Xi}_{i 12} & P-V E_{i}^{T}+\beta_{2} A_{i} V^{T}+Y_{3}^{T} & V C^{T}+Y_{4}^{T} \\
* & \widetilde{\Xi}_{i 22} & \beta_{1} V E_{i}^{T}+\beta_{2} B_{i} Z-Y_{3}^{T} & \beta_{1} V C^{T}-Y_{4}^{T} \\
* & * & d^{2} Q-\beta_{2} V E_{i}^{T}-\beta_{2} E_{i} V^{T} & \beta_{2} V C^{T} \\
* & * & -\gamma^{2} I
\end{array}\right], \\
& \widetilde{\Xi}_{i 11}=R+B_{\omega} B_{\omega}^{T}+V A_{i}^{T}+A_{i} V^{T}+Y_{1}^{T}+Y_{1}, \\
& \widetilde{\Xi}_{i 12}=Z^{T} B_{i}^{T}+\beta_{1} A_{i} V^{T}+Y_{2}^{T}-Y_{1}, \\
& \widetilde{\Xi}_{i 22}=-(1-\mu) R+\beta_{1} Z^{T} B_{i}^{T}+\beta_{1} B_{i} Z-Y_{2}^{T}-Y_{2} .
\end{aligned}
$$

Moreover, a state-feedback controller is described as $F=Z V^{-T}$.

Proof. Suppose there exist positive definite symmetric matrices $P, R, Q$, matrices $V, Y_{1}, Y_{2}, Y_{3}, Y_{4}, Z$, positive scalar $\mu$, and scalars $\beta_{1}, \beta_{2}$ satisfying (20). Multiply the above inequalities (20) by $\alpha_{i}(i=1,2, \ldots, k), \alpha_{i} \geq 0$, and $\sum_{i=1}^{k} \alpha_{i}=1$, and sum to get

$$
\left[\begin{array}{ccccc}
\widetilde{\Xi}_{11} & \widetilde{\Xi}_{12} & P-V E(\alpha)^{T}+\beta_{2} A(\alpha) V^{T}+Y_{3}^{T} & V C^{T}+Y_{4}^{T} & d Y_{1} \\
* & \widetilde{\Xi}_{22} & \beta_{1} V E(\alpha)^{T}+\beta_{2} B(\alpha) Z-Y_{3}^{T} & \beta_{1} V C^{T}-Y_{4}^{T} & d Y_{2} \\
* & * & d^{2} Q-\beta_{2} V E(\alpha)^{T}-\beta_{2} E(\alpha) V^{T} & \beta_{2} V C^{T} & d Y_{3} \\
* & * & * & -\gamma^{2} I & d Y_{4} \\
* & * & * & * & -d Q
\end{array}\right]<0
$$

where

$$
\begin{aligned}
\widetilde{\Xi}_{11}= & R+B_{\omega} B_{\omega}^{T}+V A(\alpha)^{T}+A(\alpha) V^{T}+Y_{1}^{T}+Y_{1}, \\
\widetilde{\Xi}_{12}= & Z^{T} B(\alpha)^{T}+\beta_{1} A(\alpha) V^{T}+Y_{2}^{T}-Y_{1}, \\
\widetilde{\Xi}_{22}= & -(1-\mu) R+\beta_{1} Z^{T} B(\alpha)^{T}+\beta_{1} B(\alpha) Z-Y_{2}^{T} \\
& -Y_{2}, \\
E(\alpha)= & \sum_{i=1}^{k} \alpha_{i} E_{i}, \\
A(\alpha)= & \sum_{i=1}^{k} \alpha_{i} A_{i}, \\
B(\alpha)= & \sum_{i=1}^{k} \alpha_{i} B_{i} .
\end{aligned}
$$

Then, by replacing $E, A$, and $B$ with $E(\alpha), A(\alpha)$, and $B(\alpha)$, respectively, we can obtain the desired result from Theorem 4 , easily.

\section{Numerical Example}

We consider the system (4) with

$$
\begin{gathered}
\bar{M}=\left[\begin{array}{ccc}
\delta_{1} & 0 & 0 \\
0 & 1.8 & 0 \\
0 & 0 & 1.6
\end{array}\right], \\
\bar{C}=\left[\begin{array}{ccc}
\delta_{2} & -0.6 & 0 \\
-0.6 & 1.2 & -0.6 \\
0 & -0.6 & 0.6
\end{array}\right],
\end{gathered}
$$


TABLE 1: Minimum feasible disturbance attenuation $\gamma$ by different input time delay $d$.

\begin{tabular}{lcccc}
\hline$d$ & 0.05 & 0.1 & 0.15 & 0.4 \\
\hline$[15]$ & 0.147 & 0.278 & 0.624 & - \\
{$[3]$} & 0.082 & 0.119 & 0.156 & - \\
{$[16]$} & 0.029 & 0.031 & 0.033 & 0.071 \\
Theorem 4 & 0.025 & 0.025 & 0.026 & 0.04 \\
\hline
\end{tabular}

$$
\begin{aligned}
& \bar{K}=\left[\begin{array}{ccc}
2 & -1 & 0 \\
-1 & 2 & -1 \\
0 & -1 & 1
\end{array}\right] \\
& \bar{B}=\left[\begin{array}{l}
1 \\
0 \\
0
\end{array}\right], \\
& \bar{B}_{\omega}=\left[\begin{array}{c}
0 \\
0 \\
0.1
\end{array}\right]^{T} \text {, } \\
& C=\left[\begin{array}{cccccc}
0.1 & 0.1 & 0.5 & 0 & 0 & 0 \\
0 & 0 & 0 & 0.1 & 0.1 & 0.5
\end{array}\right], \\
& \bar{x}(t)=\left[\begin{array}{lll}
\bar{x}_{1}(t) & \bar{x}_{2}(t) & \bar{x}_{3}(t)
\end{array}\right]^{T},
\end{aligned}
$$

where $\delta_{1}$ and $\delta_{2}$ are uncertain parameters. Firstly, consider the system without uncertainties, and $\delta_{1}=1.1, \delta_{2}=1.2$. This example was considered in $[3,15,16]$. Assume the control forces input time delay is $0.1 \mathrm{~s}$. By [15], we can obtain the minimum $\gamma$ (such that there exists an admissible controller) which is 0.278 . By [3], the minimum $\gamma$ is 0.119 . By [16], the minimum $\gamma$ is 0.031 . However, by Theorem 4 in this paper, it is found that the admissible controller exists even for $\gamma=0.025$ $\left(\beta_{1}=-1, \beta_{2}=-1, \mu=0.1\right)$. Thus, the less conservatism of our method is obvious. The more calculated results are presented in Table 1, which show the minimum feasible $\gamma$ for different input delay $d$. It is shown from Table 1 that the obtained results by Theorem 4 have less conservatism than those in $[3,15,16]$, obviously.

Then, let us consider vibration-attenuation performance of the closed-loop system. The corresponding disturbance input (see Figure 1) is obtained by the following signal function:

$$
\omega(t)= \begin{cases}100 \sin t, & 0 \leq t \leq 1, \\ 0, & t>1 .\end{cases}
$$

By assuming the parameters $\delta_{1}$ and $\delta_{2}$ satisfying $\delta_{1}=$ $1.1, \delta_{2}=1.2$, and choosing $\beta_{1}=-0.5, \beta_{2}=-0.1, \mu=0.1$,

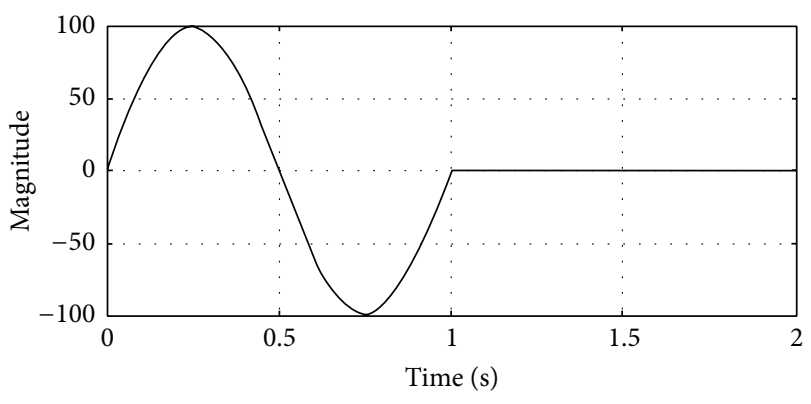

FIGURE 1: Disturbance input signal.

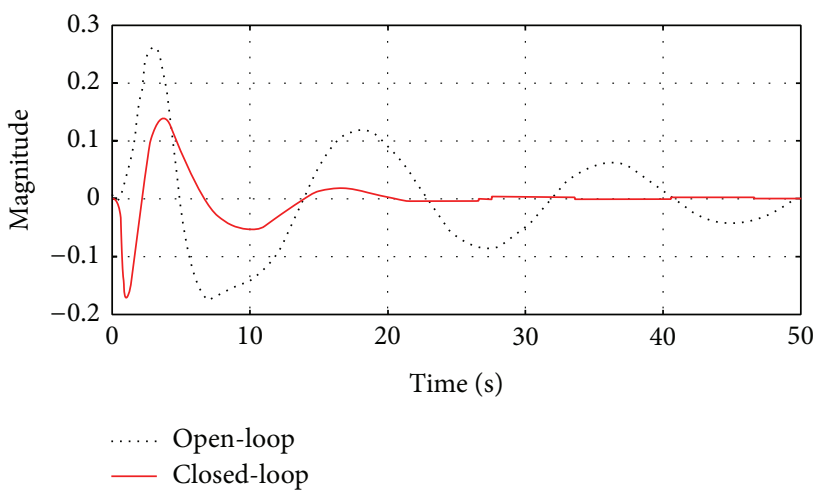

FIgURE 2: The responses of state variable $\bar{x}_{1}(t)$.

$d=0.05$, and $\gamma=0.2$, we solve LMI (8) and obtain a state feedback controller which has the following gain matrix:

F

$=\left[\begin{array}{llllll}0.2171 & -0.3699 & -0.1808 & -2.1308 & -0.2099 & -0.9866\end{array}\right]$.

For description in brevity, this state feedback controller is denoted as controller I thereafter. By taking the signal function (25) as the disturbance excitation, we can obtain the displacement responses of open-loop and closed-loop system which is composed of controller I, which are shown from Figures 2-4. It can be obtained from Figures $2-4$ that controller I is effective to attenuate the vibration of the system, obviously.

Secondly, let us consider the parameter uncertainties. By assuming the parameter uncertainties satisfying $\delta_{1} \epsilon$ $\left[\begin{array}{ll}1.1 & 1.8\end{array}\right], \delta_{2} \in\left[\begin{array}{ll}0.8 & 1.4\end{array}\right]$ and choosing $\beta_{1}=-0.5, \beta_{2}=-0.1$, $\mu=0.1, d=0.05, \gamma=0.2$, we solve LMIs (20) and obtain a robust state feedback controller which has the following gain matrix:

$$
\begin{aligned}
& F \\
& =\left[\begin{array}{lllllll}
-1.4973 & 0.2485 & 0.0501 & -2.3346 & -0.5335 & -0.3927
\end{array}\right] .
\end{aligned}
$$

For description in brevity, this state feedback controller is denoted as controller II thereafter. By taking the signal function (25) as the disturbance excitation, we can obtain the responses of $\bar{x}_{1}(t)$ in open-loop and closed-loop system which is composed of controller II, which are shown in Figure 5. The responses of $\bar{x}_{2}(t)$ and $\bar{x}_{3}(t)$ have a similar 


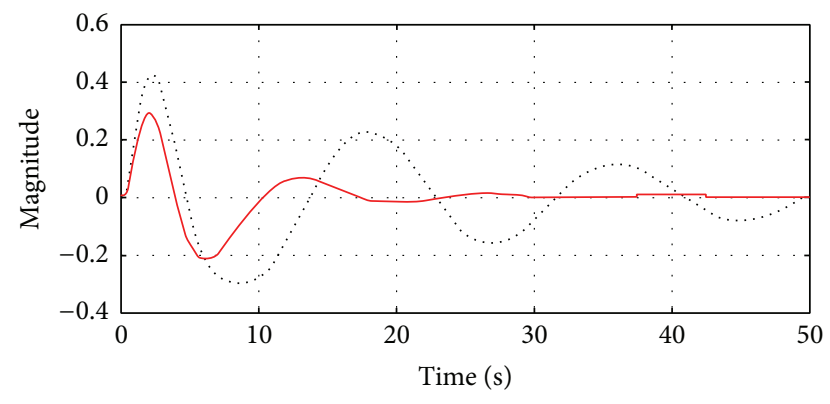

..... Open-loop

- Closed-loop

Figure 3: The responses of state variable $\bar{x}_{2}(t)$.

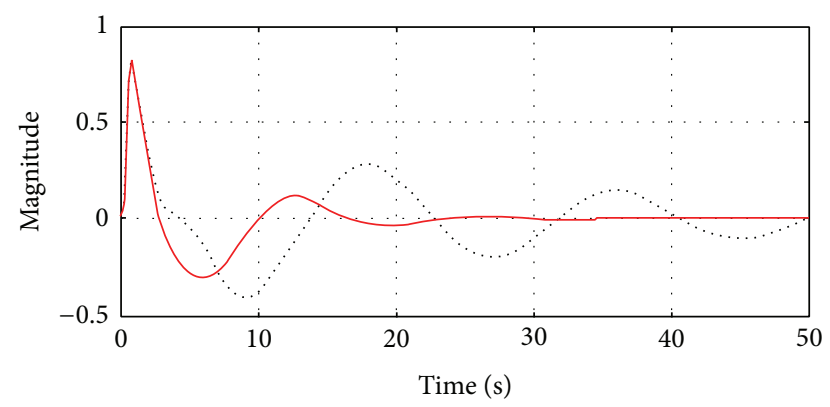

..... Open-loop

- Closed-loop

FIGURE 4: The responses of state variable $\bar{x}_{3}(t)$.

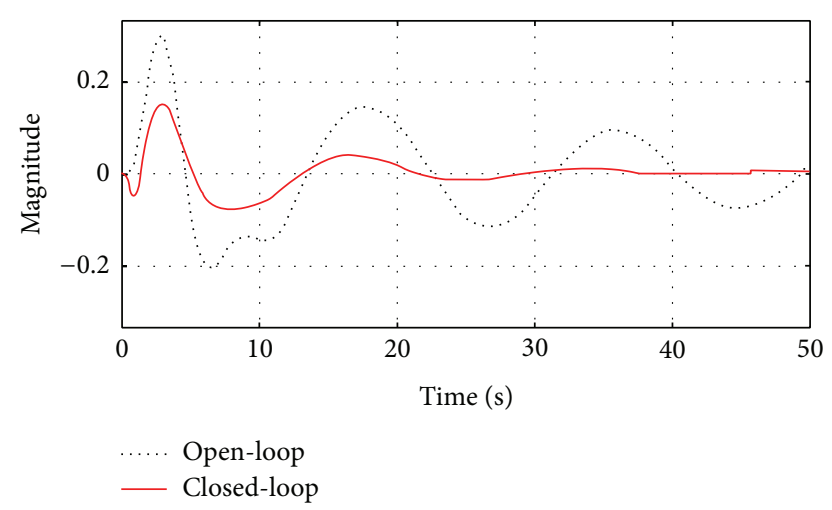

FIGURE 5: The responses of state variable $\bar{x}_{1}(t)$ in the uncertain case.

varying trend, which is omitted here for brevity. Figure 5 illuminates that designed controller II is robust to parameter uncertainties.

\section{Conclusions}

In this paper, the problems of vibration-attenuation controller design for uncertain mechanical systems with timevarying input delay are considered. First, based on matrix transformation, the mechanical system is described as a state-space model. Then, by introducing the linear varying parameters, the system model is extended to its uncertain case. Second, based on Lyapunov stability theory and LMI technique, the LMI-based condition for the system to be stabilizable is deduced. By solving these LMIs, the statefeedback control law is obtained for the closed-loop system to be stable with the performance $\|z(t)\|_{2}<\gamma\|\omega(t)\|_{2}$. In the end, numerical example is given to show the effectiveness of the proposed theorems.

\section{Conflict of Interests}

The authors declare that there is no conflict of interests regarding the publication of this paper.

\section{Acknowledgments}

This research was supported by project of National Natural Science Foundation of China (no. 61463018), project of Jiangxi Provincial Natural Science Foundation of China (no. 20151BAB207046), and project of the Natural Science Foundation of Jiangxi University of Science and Technology of China (no. NSFJ2014-K16). The authors would like to thank anonymous reviewers for their valuable comments and suggestions.

\section{References}

[1] Y. Huo and Y. Huangfu, "Synchronization of a two degreeof-freedom nonlinearly coupled mechanical systems based on terminal sliding mode control," Journal of Computational Information Systems, vol. 11, no. 2, pp. 735-742, 2015.

[2] W. Hea, C. Yang, T. Meng, and C. Sun, "Distributed control of a class of flexible mechanical systems with global constraint," International Journal of Control, 21 pages, 2015.

[3] X. Yang, H. Gao, P. Shi, and G. Duan, "Robust $H_{\infty}$ control for a class of uncertain mechanical systems," International Journal of Control, vol. 83, no. 7, pp. 1303-1324, 2010.

[4] R. Sakthivel, A. Arunkumar, and K. Mathiyalagan, "Robust sampled-data $H_{\infty}$ control for mechanical systems," Complexity, vol. 20, no. 4, pp. 19-29, 2015.

[5] P. P. Moita, J. B. Cardoso, and A. Barreiros, "Optimal design and control of mechanical systems with uncertain input," Shock and Vibration, vol. 19, no. 5, pp. 1019-1025, 2012.

[6] E. Johnson, J. Schultz, and T. Murphey, "Structured linearization of discrete mechanical systems for analysis and optimal control," IEEE Transactions on Automation Science and Engineering, vol. 12, no. 1, pp. 140-152, 2015.

[7] M. Spong, "Energy based control of a class of underactuated mechanical systems," in Proceedings of the 13th IFAC World Congress, pp. 431-435, San Francisco, Calif, USA, 1996.

[8] A. K. Sanyal and J. Bohn, "Finite-time stabilisation of simple mechanical systems using continuous feedback," International Journal of Control, vol. 88, no. 4, pp. 783-791, 2015.

[9] J. G. Romero, R. Ortega, and I. Sarras, "A globally exponentially stable tracking controller for mechanical systems using position feedback," IEEE Transactions on Automatic Control, vol. 60, no. 3, pp. 818-823, 2015.

[10] H. Du and N. Zhang, "Energy-to-peak control of seismicexcited buildings with input delay," Structural Control and Health Monitoring, vol. 14, no. 7, pp. 947-970, 2007. 
[11] A. Aleksandrov, E. Aleksandrova, and A. Zhabko, "Asymptotic stability conditions for certain classes of mechanical systems with time delay," WSEAS Transactions on Systems and Control, vol. 9, pp. 388-397, 2014.

[12] F. Weng, Y. Ding, J. Ge, L. Liang, and G. Yang, "Finite-time vibration control of earthquake excited linear structures with input time-delay and saturation," Journal of Low Frequency Noise, Vibration and Active Control, vol. 33, no. 3, pp. 245-270, 2014.

[13] Y. Liu, H. Min, S. Wang, Z. Liu, and S. Liao, "Distributed adaptive consensus for multiple mechanical systems with switching topologies and time-varying delay," Systems \& Control Letters, vol. 64, no. 1, pp. 119-126, 2014.

[14] J. Jiao and R. Zhang, "A delay decomposition approach to the stability analysis of singular systems with interval time-varying delay," Mathematical Problems in Engineering, vol. 2014, Article ID 679460, 13 pages, 2014.

[15] H. Du, J. Lam, and K. Y. Sze, " $H_{\infty}$ disturbance attenuation for uncertain mechanical systems with input delay," Transactions of the Institute of Measurement and Control, vol. 27, no. 1, pp. 37-52, 2005.

[16] F. Weng and W. Mao, "Parameter-dependent vibrationattenuation controller design for electro-hydraulic actuated linear structural systems," Earthquake Engineering and Engineering Vibration, vol. 11, no. 1, pp. 73-82, 2012.

[17] B.-L. Zhang and G.-Y. Tang, "Active vibration $H_{\infty}$ control of offshore steel jacket platforms using delayed feedback," Journal of Sound and Vibration, vol. 332, no. 22, pp. 5662-5677, 2013.

[18] Y. Ding, F. Weng, M. Tang, and J. Ge, "Active vibrationattenuation controller design for uncertain structural systems with input time-delay," Earthquake Engineering and Engineering Vibration, vol. 14, no. 3, pp. 477-486, 2015.

[19] F. Weng, Y. Ding, L. Liang, and J. Ge, "Acceleration-based vibration control for structural systems with actuator faults and finite-time state constraint," Noise \& Vibration Worldwide, vol. 46, no. 4, pp. 10-21, 2015.

[20] J. Bai and H. Su, "Robust D stabilization of singular systems with polytopic uncertainties," in Proceedings of the American Control Conference (ACC '09), pp. 3706-3709, St. Louis, Mo, USA, June 2009.

[21] Z.-G. Wu, P. Shi, H. Su, and J. Chu, "Stochastic synchronization of markovian jump neural networks with time-varying delay using sampled data," IEEE Transactions on Cybernetics, vol. 43, no. 6, pp. 1796-1806, 2013.

[22] Y.-Y. Cao, Y.-X. Sun, and C. Cheng, "Delay-dependent robust stabilization of uncertain systems with multiple state delays," IEEE Transactions on Automatic Control, vol. 43, no. 11, pp. 1608-1612, 1998. 


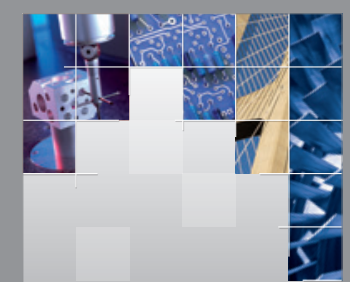

\section{Enfincering}
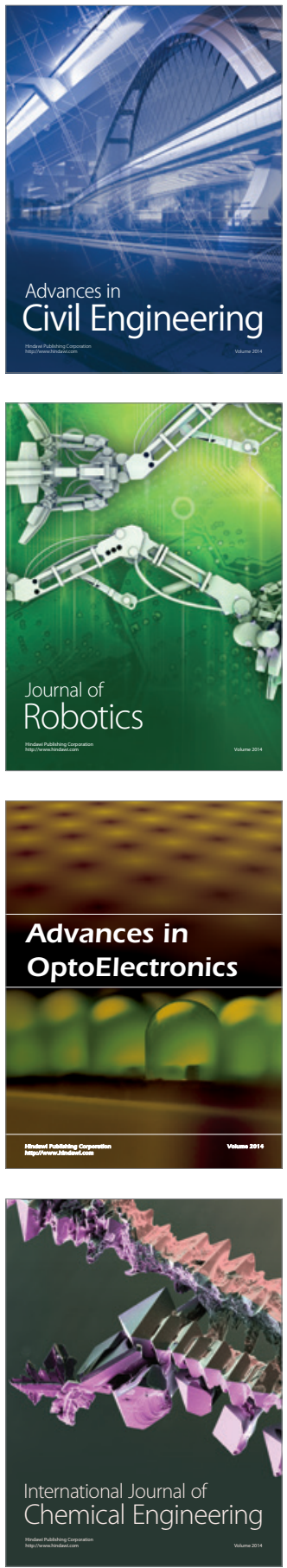

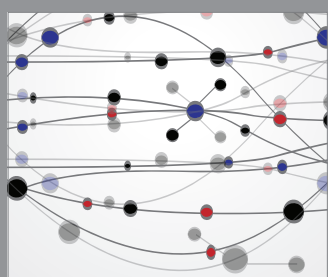

The Scientific World Journal

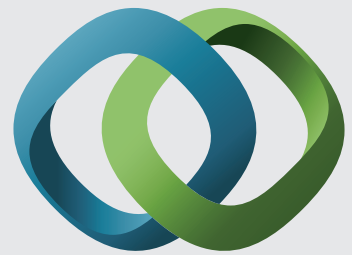

\section{Hindawi}

Submit your manuscripts at

http://www.hindawi.com
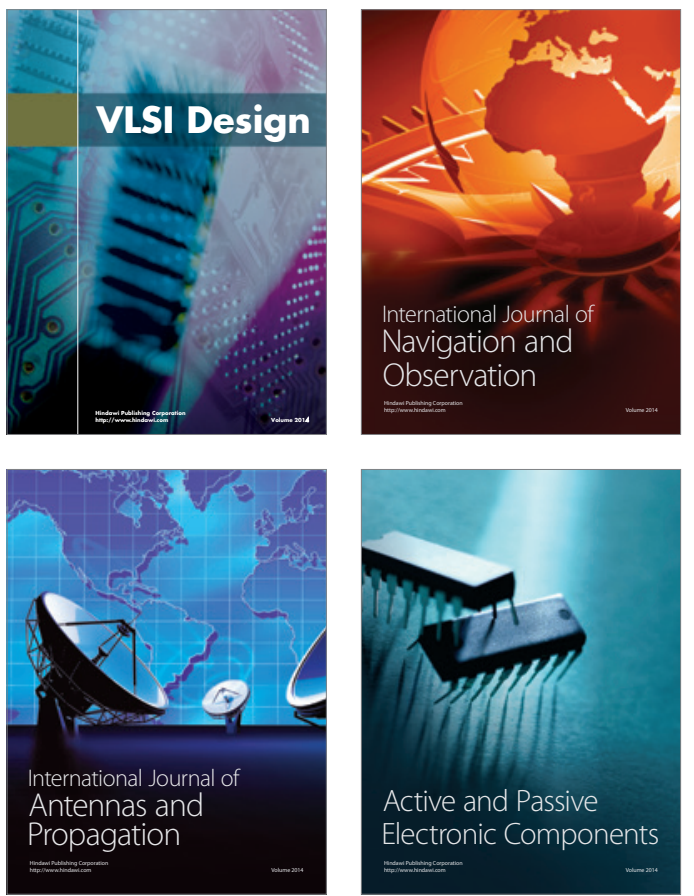
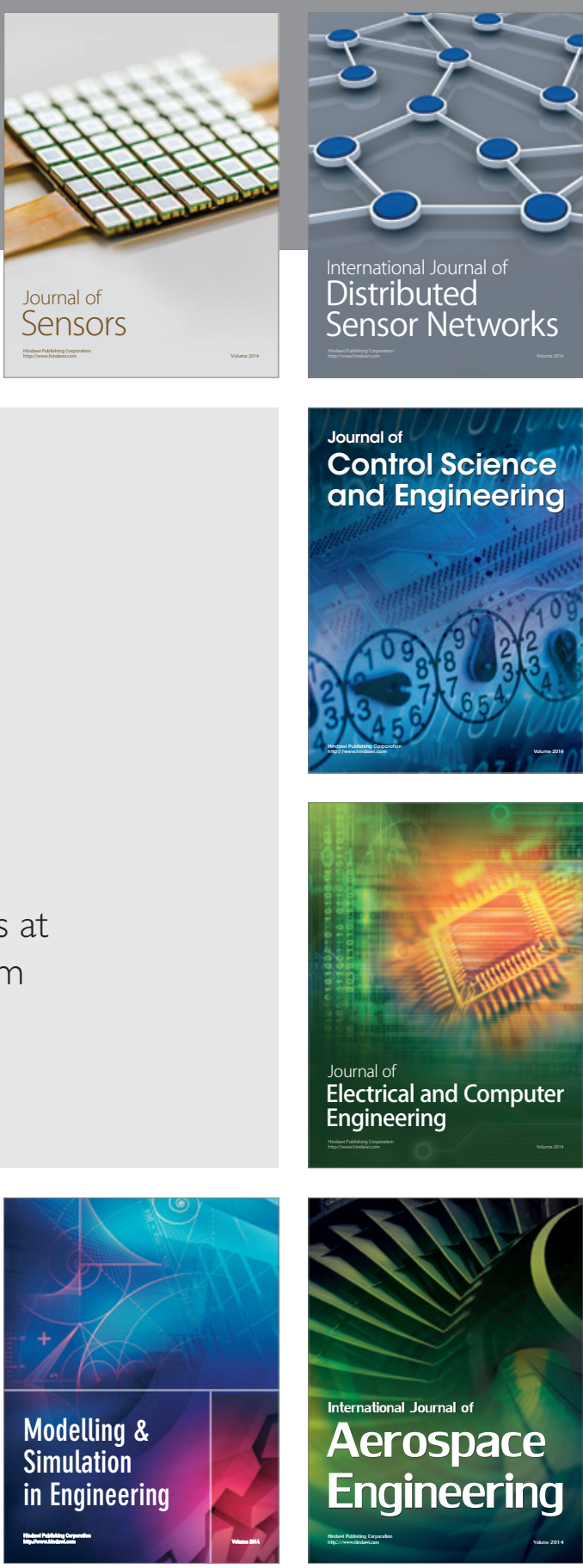

International Journal of

Distributed

Sensor Networks

Journal of

Control Science

and Engineering
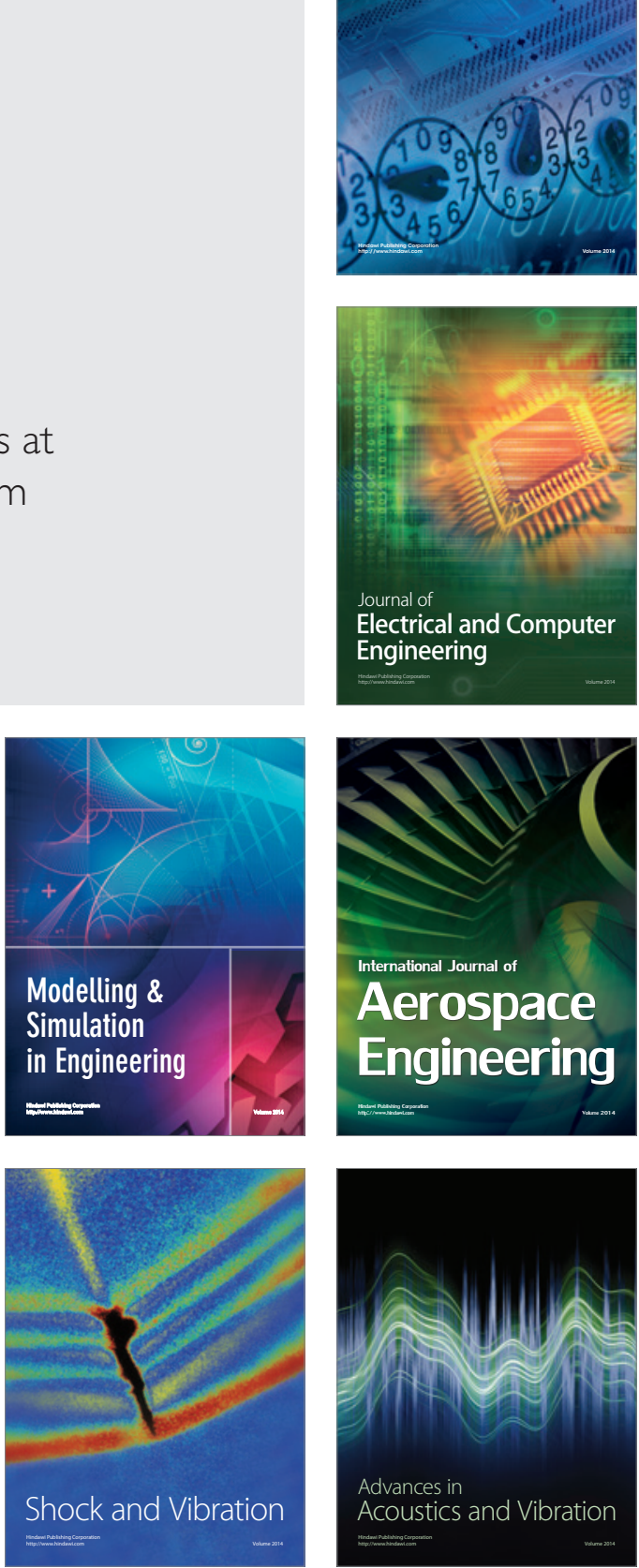